\title{
Integrating Sensors into Mechanical Structures
}

\author{
Jürgen Wilde, Thomas Fellner, Roderich Zeiser \\ University of Freiburg - IMTEK \\ Department of Microsystems Engineering \\ Laboratory for Assembly and Packaging \\ juergen.wilde@imtek.uni-freiburg.de
}

\begin{abstract}
In the case of structure-integrated sensors we regard transducers for mechanical properties like force, stress, or strain, which are mounted directly on a component. This allows much more compact solutions and reduced packaging effects compared to conventional packaging. Two main types exist. One is foil type like a strain gauge and the second is a chip-like element. Both concepts are investigated and their specifics will be further discussed. One of the principal aims of conventional packaging is to avoid mechanical stresses in a device as cross-sensitivities will affect sensitivity, offset and linearity. In the case regarded here it is the target to maximise the stress in a transducer as it is the characteristic property. Besides, sensitivity and offset must not be affected by the operating environment like temperature or humidity. As it is well-know from strain gauges, foil-type transducers are well-suited as they exhibit low inherent stiffness. Unfortunately conventional strain gauges will not be suited well for autonomous operation due to their low resistance and high power consumption. To that purpose we explored new concepts for thin-film based strain gauges. The characterisation of these sensors shows good potential for application in wireless sensing. Also assembly and packaging concepts for strain gauges with integrated circuits for instrumentation and wireless operation have been developed. In the case of chiptype transducers the principal questions of sensor mounting were investigated on SAW devices. Based on finite-element simulations different technologies for mounting chips were evaluated with respect to their influence on offset and sensitivity. Die attachment techniques like adhesive bonding, soldering, brazing, or glass bonding were regarded. Based on these results, proposals for assembly, packaging and interconnection are made.
\end{abstract}

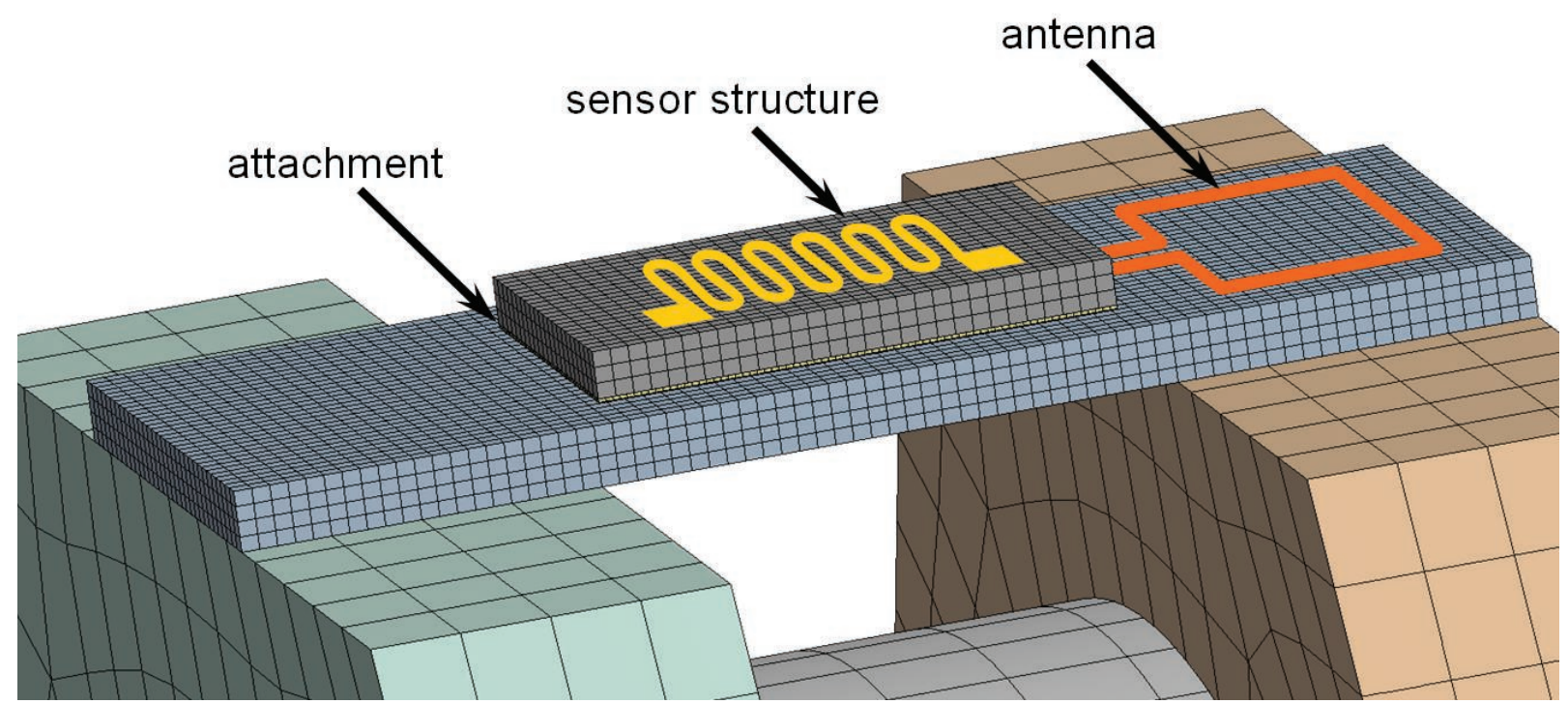

Figure 1: Finite-element model of a structure-integrated wireless force sensor exhibiting the three principal areas of interest in this investigation.

\section{Introduction}

In the past, structure-integrated sensors have already been used in mechanical engineering and in civil engineering [1]. One of the applications was load monitoring in order to analyze the actual load or the load profile or even to detect the damage to the structures of interest. Both dynamic loads and static loads have been monitored and the acquired data were used for real-time control or monitoring. The main 
types of sensors are load cells in combination with instrumentation amplifiers or strain gauges which are attached directly to the parts and which also need instrumentation amplifiers. All of these sensors are wired in order to interconnect them to the system. Bragg fibre gratings are a newer development. They consist of optical glass fibres with inscribed gratings, which are used as strain monitors. Also these need cable-bound interconnections.

In the case of mobile structures like a rotating shaft, solutions using sliding contacts have been utilized for interconnection. In recent years, the development of wireless sensor systems has made great progress. Two main areas of interest were wireless data transmission and sensor energy management. Due to these developments, more and more applications become feasible for continuous wireless sensing. If such sensors have to be integrated directly into structures in mechanical engineering applications, still several key problems are open. The principal questions to be solved are highlighted on the scheme of a structure-integrated sensor chip in Figure 1:

1. Low power consumption of sensing structures for stresses and strains. Presently, strain gauges with high resistance are not available commercially. Therefore we explore several concepts for capacitive strain gauges utilizing thin-film structures. These transducers have to be integrated with the circuitry for instrumentation, energy management and data transfer.

2. Reproducible load transfer into the transducer while avoiding cross-sensitivities. This is already a problem in conventional foil-type strain gauges. When IC sensors or SAW-devices are used, their inherent high stiffness in combination with the elasticity of the attachment material causes significant sensitivity degradation. Therefore we also address concepts where sensor chips have to be integrated into mechanical structures in this paper.

3. Besides the integration of the structures for data and energy transfer like antennas are regarded.

\section{Novel Strain Gauge Concepts}

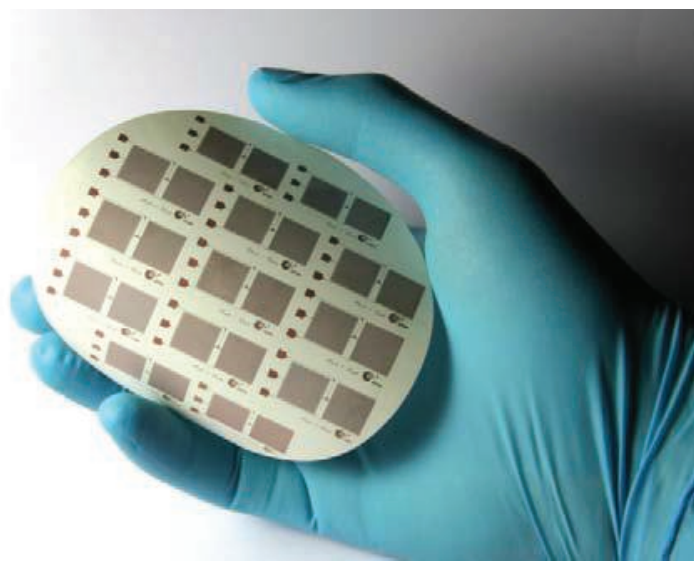

Figure 2: Capacitive strain gauge sensor structures on LCP substrate

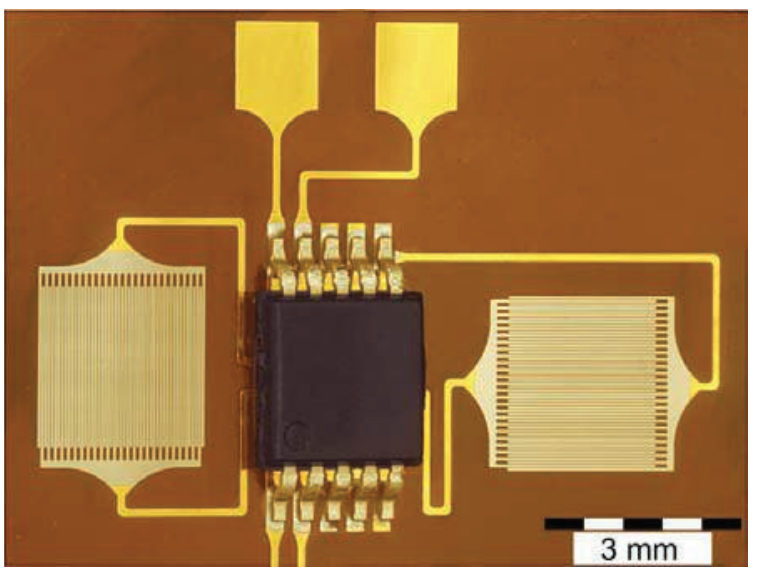

Figure 3: Capacitive strain gauge with integrated read out IC

Although strain gauges are very accurate and proven devices, their main disadvantage is their low resistance. The strain gauges in the market exhibit resistances from $500 \Omega$ up to $5 \mathrm{k} \Omega$. Using operating voltages of $5 \mathrm{~V}$ will result in dissipated powers of 5 up to $50 \mathrm{~mW}$ for the transducer alone. To that purpose, concepts for high-resistance strain gauges and also for capacitive strain gauges using thin-film materials in combination with novel carrier base materials were explored. An interesting alternative to resistive strain gauges might be sensors working through the capacitive principle. Due to the fast development of read-out ICs, it is now possible to measure capacitances with high resolution and high frequency in very low power mode. Capacitive sensors are highly dependent on the dielectric material as well as environmental influences, like temperature and humidity. These will affect the dielectric and therefore directly the sensor signal. For this reason it is important to know the cross-sensitivities on environmental influences to assess the resolution and reliability of the capacitive sensor. Several concepts for devices that measure strain with a capacitive method have been presented [2] [3] by others. In this work novel capacitive sensors are proposed, which are based on inter-digital electrodes on flexible polymer foils, and have structure heights in the nanometre range, Figures 2 and 3 . In the first step structures were analysed using a method described by Igreja [4].

A capacitor with inter-digital electrodes in one plane on a flexible substrate was chosen as sensing principle for the strain gauge. The advantages are maximum transfer of strain into the capacitor, high 
capacitance on a small area and only one process step for the in-plane electrodes. The design of these sensors was analyzed using the finite-element simulation software ANSYS. A coupled multi-physics simulation was performed that combines mechanical and electrostatic analyses.

A design for two sensors with perpendicular electrode-orientation is presented in Fig. 2. In subsequent designs, the width and clearance were optimized for capacitance and sensitivity to $45 \mu \mathrm{m}$ and $15 \mu \mathrm{m}$ respectively. There are three soldering pads where the capacitances can be connected for measurement. The ground electrodes are interconnected on a soldering pad. Sharp edges in the design are rounded to reduce the stress in these areas.

\section{Fabrication and Testing}

The materials used for substrates of the strain gauges were Polyimide (PI) and Liquid-Crystal Polymer (LCP). PI is a standard material for flexible circuits and resistive strain gauges. It exhibits outstanding properties like a high thermal and chemical stability. A disadvantage of $\mathrm{PI}$ is its high absorption of humidity with about $3 \%$ [5]. LCP is also used for HF-applications mainly because of its low dielectric losses [6]. The value of moisture absorption is about $0.03 \%$, hundred times smaller than polyimide. A special method to process polymer foils with approved thin film technologies was developed in this work. A lift-off process was used for patterning of the electrodes with structure distances of $15 \mu \mathrm{m}$. Fig. 2 shows the sensor structures on the LCP substrate before dicing.

With a universal testing machine, the sensor structures were tested with respect to their sensitivity and their cross-sensitivities against temperature and humidity in a special test chamber. The capacitances were measured with a LCR-meter in 4-wire mode. In Fig. 4, the results of a strain measurement at $23{ }^{\circ} \mathrm{C}$ in dry air with a sensor on polyimide with an electrode width of $45 \mu \mathrm{m}$ and a clearance of $15 \mu \mathrm{m}$ are presented. The output characteristic of the capacitive strain gauge is linear. The measurement reveals a strain sensitivity of $-0.658 \mathrm{pF} / \%$ and a strain coefficient $\mathrm{SC}=13,783 \mathrm{ppm} / \%$, which correspond to a gaugefactor of -1.38 .

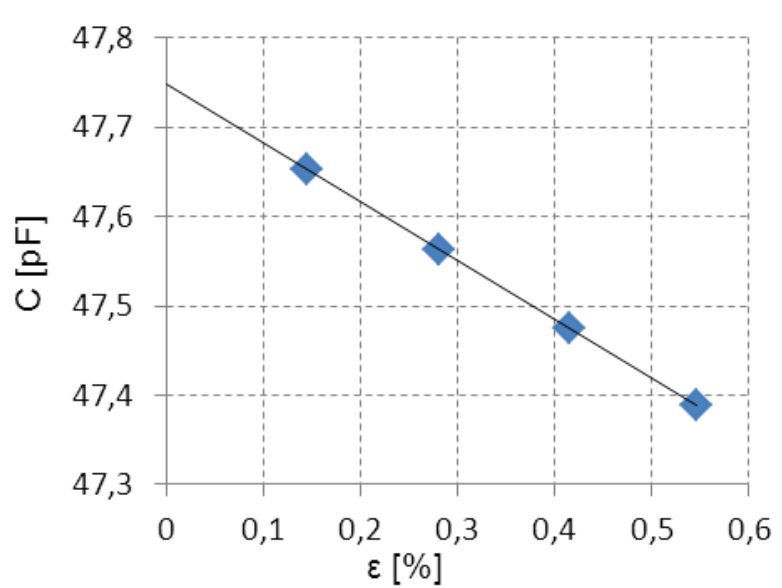

Fig. 4: Capacitance of PI sensor as a function of strain. (Note: comma is used as decimal.)

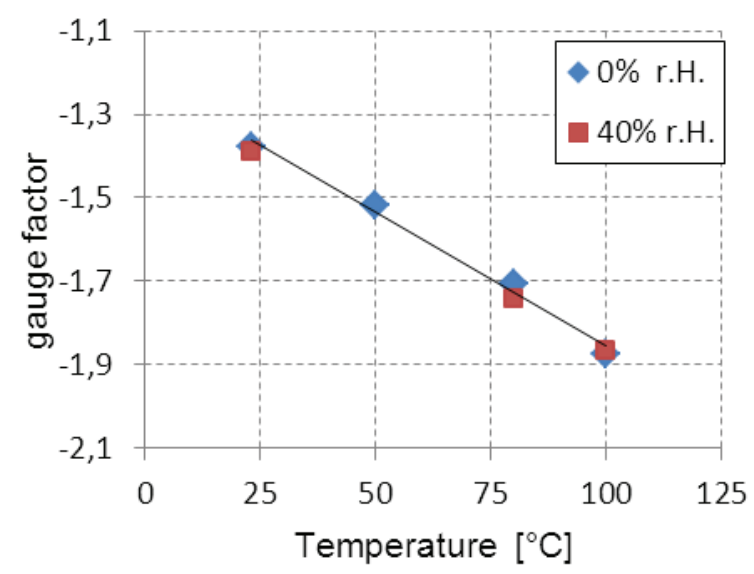

Fig. 5: Gauge factor of PI sensor as a function of temperature for $0 \%$ and $40 \%$ relative humidity

With the measuring station described it was possible to obtain the capacitance and the gauge factor of the prototype sensors at different temperatures and different humidity levels. In Fig. 5 the results for a strain gauge with polyimide substrate are presented. The sensor capacitance shows a linear dependency on humidity and temperature. In Fig. 5 the linear dependency of the gauge factor on temperature is evident. The gauge factor increases linearly with increasing temperature with a thermal coefficient of $-63.8 \mathrm{ppm} / \mathrm{K}$. This was explained by the decrease of the substrate's elastic modulus at higher temperatures. Humidity has no influence on the sensitivity of the sensor. Based on the results for the sensor $\mathrm{PI}-45 / 15$, an analytical model for the capacitance as a function of strain, temperature and relative humidity could be established:

$$
\mathrm{C}(\varepsilon, \mathrm{T}, \mathrm{H})=\mathrm{C}_{0}\left\{1+\mathrm{TC}\left(\mathrm{T}-\mathrm{T}_{0}\right)+\mathrm{HC}\left(\mathrm{H}-\mathrm{H}_{0}\right)+\mathrm{SC} \varepsilon\left[1+\mathrm{TC}_{\mathrm{SC}}\left(\mathrm{T}-\mathrm{T}_{0}\right)\right]\right\}
$$

With: TC $=2,168 \mathrm{ppm} / \mathrm{K}, \mathrm{C}_{0}=47.74 \mathrm{pF}, \mathrm{HC}=1,435 \mathrm{ppm} / \% \mathrm{rH}, \mathrm{T}_{0}=23{ }^{\circ} \mathrm{C}, \mathrm{SC}=-13,783 \mathrm{ppm} / \%, \mathrm{H}_{0}=0 \% \mathrm{rH}$, $\mathrm{TC}_{\mathrm{SC}}=-63.8 \mathrm{ppm} / \mathrm{K}$ 
For a LCP sensor which was fully encapsulated by a LCP foil on top of the sensor structures, the coefficients are $\mathrm{HC}=55 \mathrm{ppm} / \% \mathrm{rH}$ and TC $=1,958 \mathrm{ppm} / \mathrm{K}$. With these models it is possible to predict the value of the sensor capacitance if the environmental conditions and the state of strain are known. It also allows a prediction about the state of strain if the capacitance of the sensor and the environmental conditions are known.

\section{Differential Measurements}

A capacitance-to-digital converter IC was used to measure the difference of two PI-45/15 sensors directly on the substrate, Fig 2. The capacitance of one single sensor is compared with the output signal of the half bridge in Fig. 6 . During the measurement the temperature is raised by $45^{\circ} \mathrm{C}$. The capacitance of the single sensor increases by $2.5 \mathrm{pF}$ while the differential signal increases by $0.08 \mathrm{pF}$. The influence of temperature is nearly compensated. The small change of the signal is due to the disparity of the two sensors in the half-Bridge which is about $2 \%$ with respect to capacitance.

Humidity and temperature have a major impact on the output signal of the capacitive strain gauge. A compensation of the cross sensitivity for this sensor variant is necessary to ensure a correct strain determination. The influence of application of LCP foils both as sensor substrate and structure encapsulation could be further reduced down to $55 \mathrm{ppm} / \% \mathrm{rH}$.

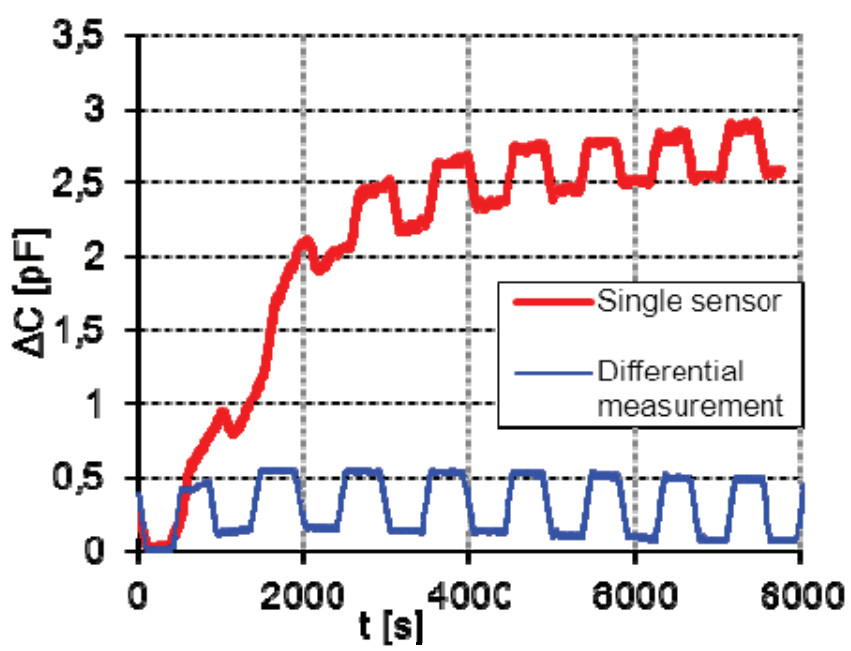

Fig. 6: Comparison of the temperature influence on the output signal of a single sensor measurement and a differential measurement on the $\mathrm{PI}$ sensor
Furthermore, it was possible to compensate both the temperature and the humidity sensitivity by differential measurement of a sensor half-bridge almost completely. The sensors on a polyimide substrate were arranged perpendicular to each other. In this case the humidity coefficient was $170 \mathrm{ppm} / \% \mathrm{rH}$ and the temperature effect could be reduced from 2,168 $\mathrm{ppm} / \mathrm{K}$ to $24 \mathrm{ppm} / \mathrm{K}$. The gauge factor of the differential sensor was -2.3.

\section{Further research}

The sensor system which has been developed on the basis of this work is shown in Fig. 3. A low power read out IC is integrated on the sensor substrate. Further steps in development will be the integration of a microcontroller, a power supply and wireless communication on the same substrate to create an intelligent, low power strain gauge for wireless application.

Also, the circuits can be optimised in order to build smart labels with humidity sensing capability [7].

\section{Load Transfer into Bulk Sensors}

The second difficulty to be addressed is concerned with the cross-sensitivities due to temperature-dependent thermal stresses. This problem is mainly affected by the sensor's stiffness as well as by the assembly technology in the mechanical structure. This is very crucial because inside machinery the temperature span can range from -40 to $125^{\circ} \mathrm{C}$. There are several influences which are known from sensor packaging that will affect the transducer's properties, such as:

- Both offset and sensitivity are temperature dependent [8].

- The mounting on the substrate material adds additional stress to the sensor due to thermalmechanical effects [9].

One of the most relevant questions in structure integration is the load transfer into the sensor. This question is especially relevant, when a stiff stress chip like a sensor IC or a SAW device is mounted onto a mechanical structure. To that purpose, we regard the case of a load sensing cell consisting of a resonant quartz device, which is mounted on an interface plate. The materials and design of the system are shown in Figure 1; the material and geometry data are listed in Table 1.

In the exhibited example the interface plate is loaded by a torque transferred from the shaft, Figure 1. This plate is subjected to two displacements between the loading points $\mathrm{dU}$. $\varepsilon=\mathrm{dU} / \mathrm{l}$ is the nominal strain in the plate.

- If a sensor is mounted on top of the plate, it will only be subjected to the nominal strain when it is coupled ideally and when its stiffness is small compared to the stiffness of the plate. In the case of 
a thicker sensor chip made out of a high modulus material like quartz or silicon, the strain in the sensor $\varepsilon_{s}$ is less than the total nominal strain $\varepsilon_{p}$ of the plane.

- A second effect is that the mounting material decouples the sensor from the plate further. This will reduce the strain in the sensing element. Only when the stiffness of the attachment material is sufficiently high, the strain is fully taken up by the sensor.

\begin{tabular}{|l|c|c|c|}
\hline Function & Material & $\begin{array}{c}\text { Coefficient of Thermal } \\
\text { Expansion }(\mathrm{CTE}) \alpha\end{array}$ & Young's Modulus \\
\hline Sensor & Quartz & $12 \cdot 10^{-6} / \mathrm{K}$ & $200,000 \mathrm{MPa}$ \\
\hline Base plate & Steel & $12 \cdot 10^{-6} / \mathrm{K}$ & $210,000 \mathrm{GPa}$ \\
\hline Bond Material & Glass, polymer, metal & & 1,000 to $300,000 \mathrm{GPa}$ \\
\hline
\end{tabular}

Table 1: Material data for the simulation of the attachment of structure-integrated force sensors

There are many possibilities to mount a sensor onto a substrate. The alternatives are adhesive bonding, soldering, brazing, welding, or glass bonding. A number of these are listed in Table 2 with their respective advantages and drawbacks. Also in the case of a sensor, typical stresses will develop in the device as a result of the different CTEs $\alpha$. The developed stress is typically computed via a formula according to

$$
\sigma_{s}=C \cdot E \cdot \varepsilon_{s}=C \cdot E_{s} \cdot \Delta \alpha \cdot \Delta T=C \cdot E_{s} \cdot\left(\alpha_{s}-\alpha_{p}\right) \cdot\left(T_{s f}-T_{u s e}\right)
$$

In this formula (2), $\mathrm{E}$ is the elastic modulus, $\alpha$ is the CTE and T is the temperature. The index sf denominates the stress-free temperature and the index use the temperature of the application. It has been demonstrated in the case of pressure sensors and magnetic field sensors that even if the behaviour of the materials is strongly temperature dependent, it can still be modelled. In this case the appropriate temperature and time dependent materials data must be available.

Because there are many geometry influences, the case of a structure-integrated sensor was investigated using the finite-element method. The details of the simulation are found in Table 2 and in Figure 7 together with the results. In the simulations, the influence of Young's Modulus of the attachment material on the coupling factor of a structure-integrated sensor was computed systematically. Materials and geometry data according to Table 1 were used.

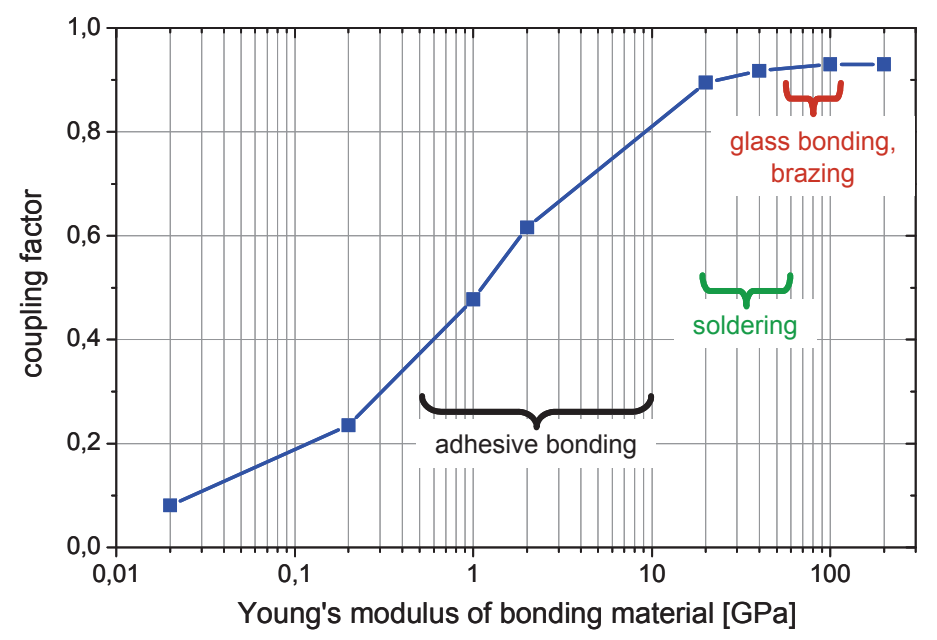

Figure 7: Influence of Young's Modulus of the attachment material on the strain coupling factor of a structure-integrated quartz sensor mounted on a steel substrate.

In the adhesive bonding the range of elastic modulus ranges from several hundred MPa up to $20 \mathrm{GPa}$ [10]. Unfortunately even a single adhesive can cover a respective range if the temperature is changed from below the glass transition temperature to a value significantly above $T_{g}$. This will lead to a situation where in the case of a stiff sensor chip, the strain in the sensor $\varepsilon_{s}$ is significantly less than that of the total nominal strain $\varepsilon_{p}$ of the plane at elevated temperatures. Consequently the coupling factor will range between $\mathrm{k}=0.3$ and $\mathrm{k}=0.9$ over the temperature range. So the standard process for sensor mounting will 
not be well-suited in cases where the structural loads must be transferred correctly. The solder material seems to be suited better, due to the higher elastic modulus. It has been proven, that as solder material creeps, a reduction of the transferred stresses as well as non-linearity in offset and sensitivity must be expected, Table 2 .

\begin{tabular}{|c|c|c|c|}
\hline Attachment process & Advantages & Disadvantages & Process parameter \\
\hline Adhesive bonding & $\begin{array}{l}\text { - Mature \& versatile process } \\
\text { - No special surfaces } \\
\text { - Low thermal stress }\end{array}$ & $\begin{array}{l}\text { - Highly temperature } \\
\text { dependent properties } \\
\text { - Material creeps }\end{array}$ & $\begin{array}{l}\text { - } \mathrm{T}_{\text {process }} \geq \mathrm{RT} \\
\text { - Seconds to hours }\end{array}$ \\
\hline Soldering & $\begin{array}{l}\text { - Good process control } \\
\text { - Low thermal stress }\end{array}$ & $\begin{array}{l}\text { - Material creeps } \\
\text { - Solderable surfaces }\end{array}$ & $\begin{array}{l}\text { - } \mathrm{T}_{\text {process }} \geq 220^{\circ} \mathrm{C} \\
\text { - Few seconds }\end{array}$ \\
\hline Brazing & $\begin{array}{l}\text { - Very temperature-stable } \\
\text { - High stiffness, no creep }\end{array}$ & $\begin{array}{l}\text { - High temperatures } \\
\text { - Brazeable surfaces }\end{array}$ & $\begin{array}{l}\text { - } \mathrm{T}_{\text {process }} \geq 700{ }^{\circ} \mathrm{C} \\
\text { - Few seconds }\end{array}$ \\
\hline Glass bonding & $\begin{array}{l}\text { - Very temperature stable } \\
\text { - No creep relaxation } \\
\text { - No special surfaces }\end{array}$ & $\begin{array}{l}\text { - High thermal stress in } \\
\text { bonding }\end{array}$ & $\begin{array}{l}\text { - } \mathrm{T}_{\text {process }} \geq 300{ }^{\circ} \mathrm{C} \\
\text { - Minutes }\end{array}$ \\
\hline
\end{tabular}

Table 2: Evaluation of different methods for the attachment of structure-integrated force sensors

Brazing is a process like soldering, but at temperatures above $450{ }^{\circ} \mathrm{C}$ and in reality above $700{ }^{\circ} \mathrm{C}$. These temperatures are too excessive to be applied to the sensitive sensor chips, Table 2 . Further processes for chip attachment have been reported like reactive soldering, glass bonding or low-temperature sintering. By means of such novel high-modulus materials and adapted processes reduced thermal influences on the sensors and simultaneously high temperature stability are to be expected, Table 2. Unfortunately, all of these prospective processes are still in the research phase and must be evaluated thoroughly.

\section{References}

[1] C. Gradolph, A. Friedberger, T. Ziemann, G. Müller, J. Wilde, "Rotor blade integrated sensor for monitoring of BVI caused pressures fluctuations", Asia-Pacific Conference Of Transducers And MicroNano Technology, Singapore, Republic of Singapore, (2006)

[2] Ryosuke Matsuzaki, "Wireless flexible capacitive sensor based on ultra-flexible epoxy resin for strain measurement of automobile tires", Sensors and Actuators, A 140 (2007) pp. 32-42

[3] J. Aebersold, "Design, modeling, fabrication and testing of a MEMS capacitive bending strain sensor", Computers and Typesetting, Vol. 34 Addison-Wesley, 2006

[4] Rui Igreja, C.J. Dias, "Analytical evaluation of the interdigital electrodes capacitance for a multi-layered structure", Sensors and Actuators, A 112 (2004) pp. 291-301

[5] J. Melcher, "Dielectric effects of moisture in polyimide", IEEE Transactions on Electrical Insulation, Vol. 24 (1989) pp. 31-38

[6] Zhang Xia, "Development of sop module technology based on Icp substrate for high frequency electronics applications", Electronics Systemintegration Technology Conference, (2006) pp. 118-125 [7] A. Oprea, N. Bârsan, U. Weimar, M.-L. Bauersfeld, D. Ebling, J. Wöllenstein, "Capacitive humidity sensors on flexible RFID labels", Sensors and Actuators B: Chemical, Volume 132, Issue 2, 16 June 2008, pp. 404-410

[8] E. Deier, J. Wilde, "Materials-based Modelling of Packaging Effects on the Accuracy of Micro-Sensors" Proceedings Sensor 2009 14th Int. Conf., vol. 2, Nuremberg, Germany, 2009, pp. $283-288$

[9] D. Pustan, E. Rastiagaev, J. Wilde, 'In Situ Analysis of the Stress Development during Fabrication Processes of Micro-Assemblies", 59th ECTC, San Diego, California, USA, (2009), pp. 117 - 124

[10] S. Fischer, J. Wilde, E. Deier, E. Zukowski "Influence of materials data on the performance modelling in the design of MEMS packages", 9th International Symposium On Advanced Packaging Materials,

Atlanta, USA, 2004, pp. 57-62 